\title{
Prospects of the Hard X-Ray Instrument POLAR to Measure Polarization of Solar
}

\section{Flares}

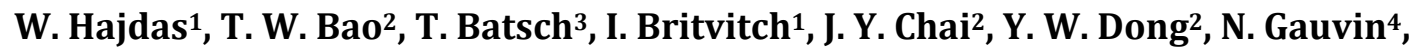
M. N. Kong ${ }^{2}$, R. Kramert ${ }^{1}$, C. Lechanoine-Leluc ${ }^{4}$, Lu Li ${ }^{2}$, J. T. Liu ${ }^{2}$, X. Liu ${ }^{2}$, R. Marcinkowski ${ }^{1}$, S. Orsi' ${ }^{4}$, M. Panicca ${ }^{4}$, M. Pohl ${ }^{4}$, N. Produit ${ }^{4}$, D. Rapin' ${ }^{4}$, A. Rutczynska ${ }^{4}$, D. Rybka ${ }^{1,3}$, H. L. Shi ${ }^{2}$,

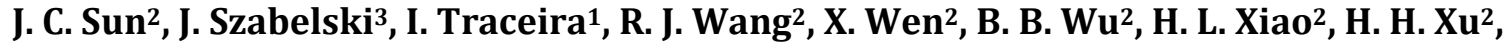
Li Zhang', L. Y. Zhang'2, S. N. Zhang'2, Y. J. Zhang'2, A. Zwolinska ${ }^{3}$

\footnotetext{
${ }^{1}$ Paul Scherrer Institute, Villigen, Switzerland

${ }^{2}$ Institute of High Energy Physics, Beijing, China

${ }^{3}$ National Centre for Nuclear Research, Swierk, Poland

${ }^{4}$ University of Geneva, Geneve, Switzerland

Email: wojtek.hajdas@psi.ch
}

Received 19 November 2014

\begin{abstract}
In-depth studies of solar flares emissions and energy releases include analyses of polarization data. Polarization gives clear information about mechanisms and processes leading to electron acceleration and photon production. Despite of many past attempts, the key energy range of hard $\mathrm{X}$-rays was only rarely explored and results were inconclusive. To large extend it was due to greater instrumental complications. Currently several novel polarimeters are either to be employed or under constructions for both balloon and satellite based observations. The novel hard $\mathrm{X}$-ray polarimeter POLAR is an instrument developed by a collaboration between Switzerland, China and Poland. It is primarily designed for high accuracy polarization measurements from the prompt photon emissions of the gamma-ray bursts. The satellite orientation and instrument pointing direction make it also capable for precise measurements of polarization in solar flares. The instrument should fly in near future onboard of the Chinese Space Station TG2.
\end{abstract}

\section{Keywords}

X-Rays, Solar Flares, Instrumentation, Polarimeters, Modeling, Qualification

\section{Introduction}

The linear polarization of solar flare photons with energies in the hard X-ray spectral range is of highest imporHow to cite this paper: Hajdas, W., Bao, T.W., Batsch, T., Britvitch, I., Chai, J.Y., Dong, Y.W., Gauvin, N., Kong, M.N., Kramert, R., Lechanoine-Leluc, C., Li, L., Liu, J.T., Liu, X., Marcinkowski, R., Orsi, S., Panicca, M., Pohl, M., Produit, N., Rapin, D., Rutczynska, A., Rybka, D., Shi, H.L., Sun, J.C., Szabelski, J., Traceira, I., Wang, R.J., Wen, X., Wu, B.B., Xiao, H.L., Xu, H.H., Zhang, L., Zhang, L.Y., Zhang, S.N., Zhang, Y.J. and Zwolinska, A. (2015) Prospects of the Hard X-Ray Instrument POLAR to Measure Polarization of Solar Flares. Journal of Applied Mathematics and Physics, 3, 272-278. 
tance in searching for explanation of its acceleration and emission mechanisms. Physical modeling requires macroscopic systems with complex magnetic fields, high, multipart temperature and density fields of plasma, and very large, not isotropic fluxes or currents of energetic electrons. Moreover the tight constraints for process timing and acceleration rates of electrons as well as the total energy emission with directionality and spectral features included, must also be kept. Accelerated electrons are revealed by bremsstrahlung and Compton scattering (albedo effect). At presents one considers basically three different energization methods: large scale direct electric fields, stochastic with gyro-resonance and "transit-time" acceleration as well as bulk heating thermal processes. The review of literature about them along with results coming from one of the recent modeling approaches based on Monte Carlo simulations of the photon transport can be found in [1]. Several measurements of the polarization in solar flares have been already attempted although usually the results were met with a grain of doubt. The method used for polarization determination was commonly based on the Compton scattering. The Klein-Nishina equation describing Compton scattering process shows sensitivity to the direction of the photon polarization vector-see Equation (1) and Figure 1. $E$ and $E^{\prime}$ indicate initial and final photon energy, $\Theta$ is the scattering angle and $\eta$ is the angle between polarization vector and the scattering plane.

$$
\frac{d \sigma}{d \Omega}=\frac{r_{0}^{2}}{2}\left(\frac{E^{\prime}}{E}\right)^{2}\left(\frac{E}{E^{\prime}}+\frac{E^{\prime}}{E}-2 \sin ^{2} \theta \cos ^{2} \eta\right)
$$

Measurement of the cross section as a function of the angle $\eta$ provides a modulation curve with an azimuth distribution of polarized photos. It allows for determination of two observables: polarization level $\Pi$ and angle $\eta_{0}$. The measurement quality clearly depends on specific parameters of the polarimeter and in particular on its modulation factor for $100 \%$ polarized photons- $\mu_{100}$. Another parameter combining instrument features with observational conditions is the minimum detectable polarization (Equation (2)).

$$
\Pi_{\text {minDet }}=\frac{n_{\sigma}}{\mu_{100} S_{f} \epsilon A_{p}} \sqrt{\frac{S_{f} \epsilon A_{p}+B_{r}}{T}}
$$

where: $n_{\sigma}$ is level of accuracy, $T$-observing time, $S_{\digamma}$-source flux, $B_{r}$-background rate, $\varepsilon$-detector efficiency and $A_{p}$-detection area. Unclear polarization results in the past were related with difficulties to optimize Equation (2) with respect to e.g. effective area, modulation factor or background levels.

\section{Polarimeters and Solar Flare Observations}

\subsection{Previous Instruments and Data}

Very first measurements indicating polarization in solar flares at energies below $15 \mathrm{keV}$ came from the Intercosmos satellites. One of the first instruments dedicated fully for polarization studies was the polarimeter for the SRG mission (that however newer flew). It had the Bragg crystal and Lithium scatterer polarimeter. Its more advanced implementations were placed on two Russian CORONAS spacecraft. The CORONS-F with observations below $100 \mathrm{keV}$ could provide mainly only upper limits: $\Pi<40 \%$ while its advanced successor CORONAPHOTON failed after few months. The Ge-spectrometer of the RHESSI mission could also work as a polarimeter. In the passive mode its five, out of nine, detectors measured photons Compton-scattered in the Be-block placed in between them. In the active mode the coincidences between two closed Ge-detectors were utilized. This mode was efficient in the energy range from about $200 \mathrm{keV}$ up to $1 \mathrm{MeV}$. Two large flares were analyzed using RHESSI by Boggs [2] though relative errors were reaching 50\%. Suarez et al. [3] could analyze seven
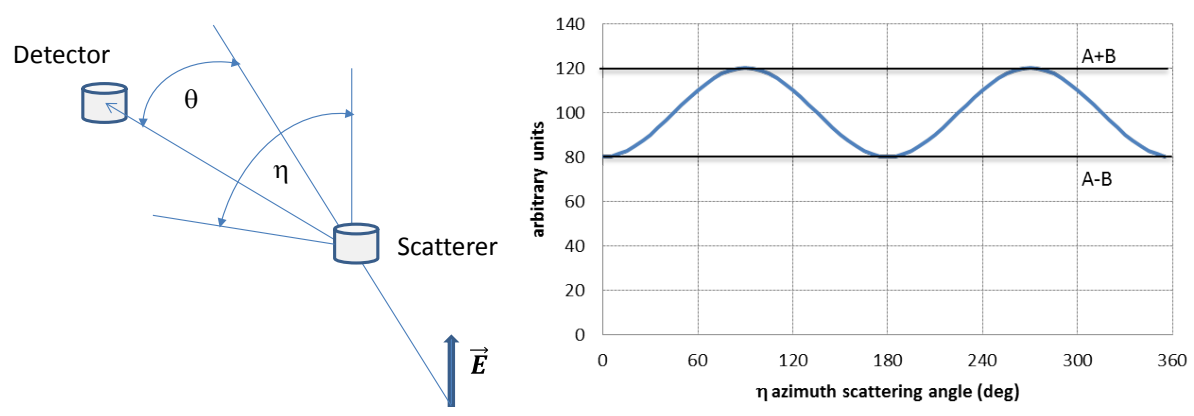

Figure 1. Scattering experimental setup (left) and example of the modulation curve (right). 
flares based on the RHESSI data. One could again only establish the upper polarization limits: $\Pi<54 \%$. The main reason for measurement scarcity is due to large experimental obstacles resulting in either low statistics of collected events or large uncertainties from very small signal to background ratio. For example, the RHESSI mission despite of quite large modulation factor $\mu_{100}$ had very small effective area $\varepsilon A_{p}$ of just a few $\mathrm{cm}^{2}$. Especially in the passive mode the background in the detectors made proper extraction of the signal extremely difficult. Please see a larger review of these and other polarization instruments and measurement results in [4].

\subsection{New Developments}

Very fast and dynamic development of new polarimeters with testing of new technologies and design concepts has started just in the last decade. New instruments have features allowing for much better and accurate polarimetry. The techniques used made it possible to enlarge the active area by orders of magnitude. At the same time the background suppression became much more effective. In addition, it became a routine that each instrument is properly modeled and its modulation factor is experimentally verified. Wider review is given in [5] while here we discuss two characteristic examples. The Polarized Gamma-ray Observer POGO is shown schematically in Figure 2 (left). It has a large area $\left(\mathrm{A}_{\mathrm{tot}} \approx 1 \mathrm{~m}^{2}\right)$, large modulation factor $\left(\mu_{100} \approx 25 \%\right)$ and it is optimized for long balloon flights. Its $400 \mathrm{BGO}$ detectors are placed at the bottom of the phoswich fast and slow scintillator counters forming walls of the well. The instrument is optimized for energy range from $25 \mathrm{keV}$ to $200 \mathrm{keV}$ and for point-like observations with a good level of background suppression [6]. The Medium Energy Gamma-Ray Astronomy Experiment MEGA showed in Figure 2 (right) is an example of the polarimeter with particle tracking features. It consists of 32 layers of double-sided Si-microstrip detectors $(36 \mathrm{~cm} \times 36 \mathrm{~cm})$ and a calorimeter made of the three-dimensional CsI(Tl)/PIN diode arrays. MEGA operates between $0.4 \mathrm{MeV}$ and $50 \mathrm{MeV}$ and the polarization measurements with the Compton scattering are feasible up to $5 \mathrm{MeV}$. The $\mu_{100}$ values at $0.7 \mathrm{MeV}$ is equal to $19 \%$ [5]. MEGA has a large field of view of 130 and effective anticoincidence shielding. It can be installed as either a satellite or a balloon polarimeter.

\section{POLAR Instrument}

\subsection{Basic Features}

POLAR instrument was developed with the goal to measure linear polarization of the gamma-ray bursts (GRB) [7]. Its design features were optimized based on lessons learnt from the previous polarimeters e.g. RHESSI or INTEGRAL. POLAR has been tuned to measure polarization in the energy range from $50 \mathrm{keV}$ to $500 \mathrm{keV}$. This range is also the most interesting for studies of the polarization in solar flares. The measurements utilize the classic Compton mechanism with photon scattering between two active detector elements (bars). The main feature distinguishing POLAR from other polarimeters is that its detection system consists of solely very light scattering elements namely 1600 plastics scintillator bars. It makes a very efficient instrument with respect to its polarization figure of merit. While the modulation factor is at least as good as in other constructions, its effective area for polarization measurement is much larger, e.g. almost two orders of magnitude larger than RHESSI. Thus, it is also much better suited for solar flares polarization studies. The scintillators are grouped into blocks of $8 \times 8$ bars $\left(6 \times 6 \times 180 \mathrm{~mm}^{3}\right)$ providing their light output to 25 multi-anode $(8 \times 8)$ photo-multipliers. The 50
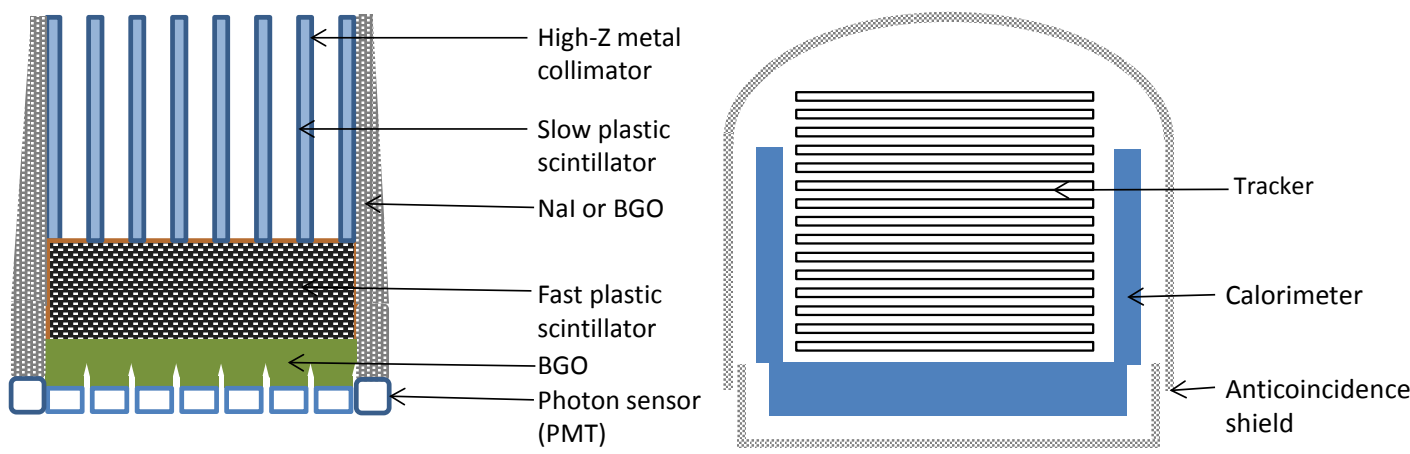

Figure 2. Examples of new polarimeters: Polarized Gamma-Ray Observer POGO (left) and Medium Energy Gamma-Ray Astronomy Experiment MEGA (right). 
keV low energy threshold for polarization detection requires the effective instrumental threshold to be as low as about $5 \mathrm{keV}$ and puts strong constraints for the scintillating photons collection quality. The azimuthal distribution of the scattered photons in the scintillator matrix provides the information on the source polarization. It requires coincidence measurements between (at least) two detection elements in which the photon is either scattered or absorbed. This task is realized with a miniaturized readout system based on the analogue-digital ASIC (VATA64-TAP3 from IDEAS) and low power FPGA handling the signal detection procedure. They constitute so called front-end electronics (FEE) and process the signals from 64 channels of one MAPMT. The ASIC provides fast trigger output with information of how many bars received the pulse. It enables to make decision about further event preprocessing and analogue readout of pulse amplitudes stored in a buffer. This decision is made by the Central Task Processing Unit (CT) which collects information from 25 front-ends, gathers the data and send to the spacecraft for further downlink to Earth. The principle of the POLAR detection of photon scattered between tow bars is shown in Figure 3 (left). The schematic view of the full POLAR and the extended view of components in one single detection module is shown in Figure 3 (center and right). The main POLAR parameters are given in the Table 1.

\subsection{Performance Modeling}

For proper determination of the POLAR performance an intensive modeling of the instrument was initiated already at the start of the project. The full polar mass model was constructed with very accurate description of the
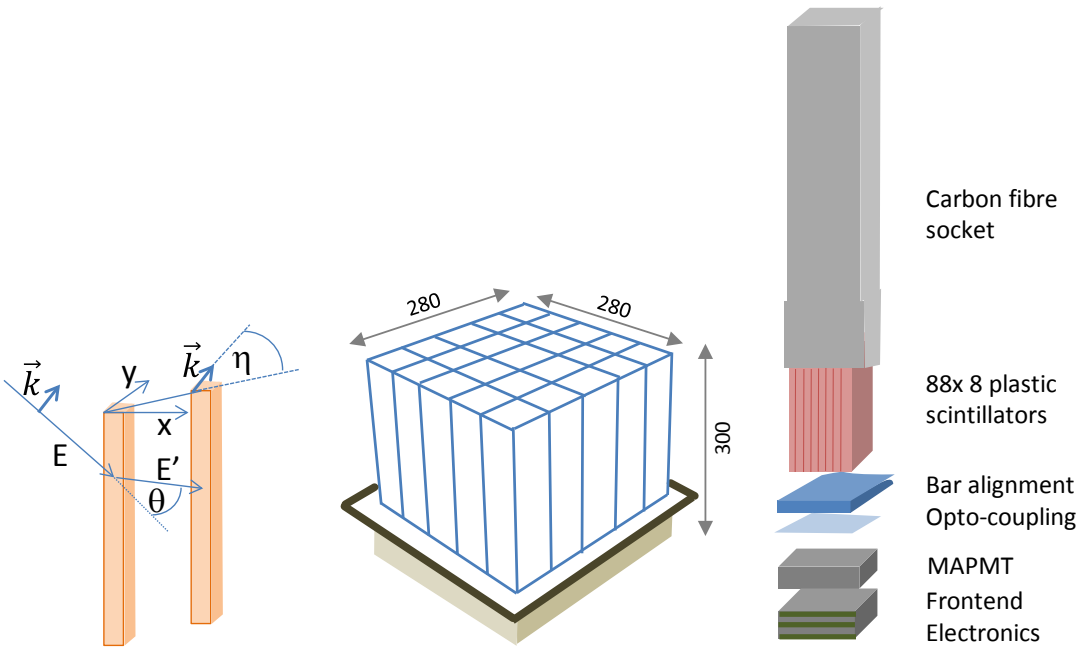

Figure 3. Detection principle of the photon with initial energy E and polarization vector $\vec{k}$ (left); POLAR schematic view 25 modules (center); Expanded view of the single detection module with $8 \times 8$ plastic scintillators (right).

Table 1. POLAR main features.

$\begin{array}{cc}\text { keV/Energy range } & 50-500 \\ \text { Field of view FOV } & 1 / 3 \text { of the sky } \\ \text { MDP/\% } & \sim 5 \\ \text { GRB and flares detected } & \text { Several tens per yea } \\ \text { Mass } / \mathrm{kg} & 33 \\ \text { Size/cm } & \\ \text { Power/W } & 45 \times 45 \times 45 \\ \text { Mission lifetime/y } & 50\end{array}$


whole detector systems. Special care was taken for a proper description of the light transport in the plastic scintillator bars. The assumptions utilized both the results of experimental tests of the reflecting foils as well as new features in the GEANT4 Monte Carlo simulation code [8]. The principal parameters of POLAR instrument were computed including its modulation factor and active area. Two examples showed in the Figure 4 present both parameters as a function of the initial photon energy for incoming direction averaged in the angular range of polar angles $\theta$ from $0^{\circ}$ to $45^{\circ}$. This range is also representative for future measurements of the polarization in solar flares. Further modeling was devoted to as broad as possible background studies. It included rough implementation of the spacecraft mass model needed for estimation of such sources of the background as positrons and neutrons. Further sources included space electrons, X-ray diffuse background as well as discrete X-ray sources such as the Crab nebula. The results are presented in the Figure 5 and compared with typical GRB or SF signal.

\subsection{Qualification and Flight Model}

POLAR went thought all steps of the space project development from the Demonstration Model, through the Qualification Models (QM) up to manufacturing of the Flight Model (FM) and Flight Model Spare (FMS). To assure full functionality in conditions of harsh environment the major subsystems of POLAR were made redundant. This way the Central Task Processing Unit as well as the Low and High Voltage Power Supply were doubled. For the FFE units one can switch the defective channels or even the whole unit off with only slight decrease of the performance. Special measures were taken to protect FEE against radiation effects such as Single Event Latch-ups. On the mechanical side POLAR was equipped with custom-made bumpers for fixation to the spacecraft body. They safeguard the instrument against disastrous vibration and shock effects during the launch. Shocks might be extremely dangerous e.g. to the MAPMT. The QM is showed in the Figure 6 (left). In the year 2013 and 2014 it underwent the full series of qualification procedures and calibration runs aimed to prove that POLAR fulfils its requirements and will be able to operate safely in space during the nominal mission duration of up to 3 years. The results of the modulation factor $\mu_{100}$ measurements and verification are presented in the
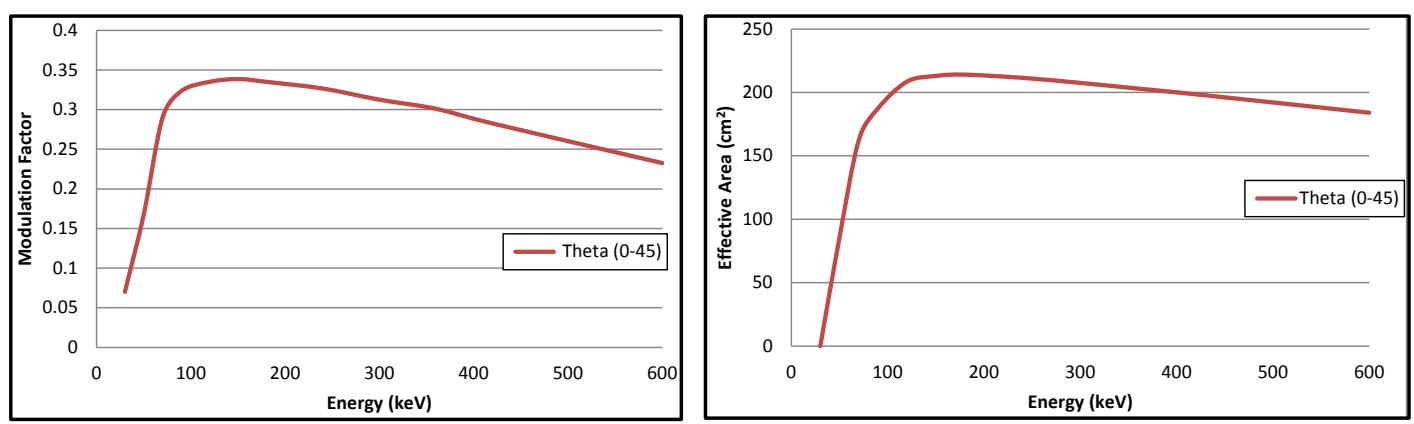

Figure 4. Modulation factor $\mu_{100}$ (left) and active detector area $A_{\text {eff }}$ (right) as a function of photon energy. Curves show results of Monte Carlo simulations for photon incoming polar angles $\theta$ between $0^{\circ}$ and $45^{\circ}$.
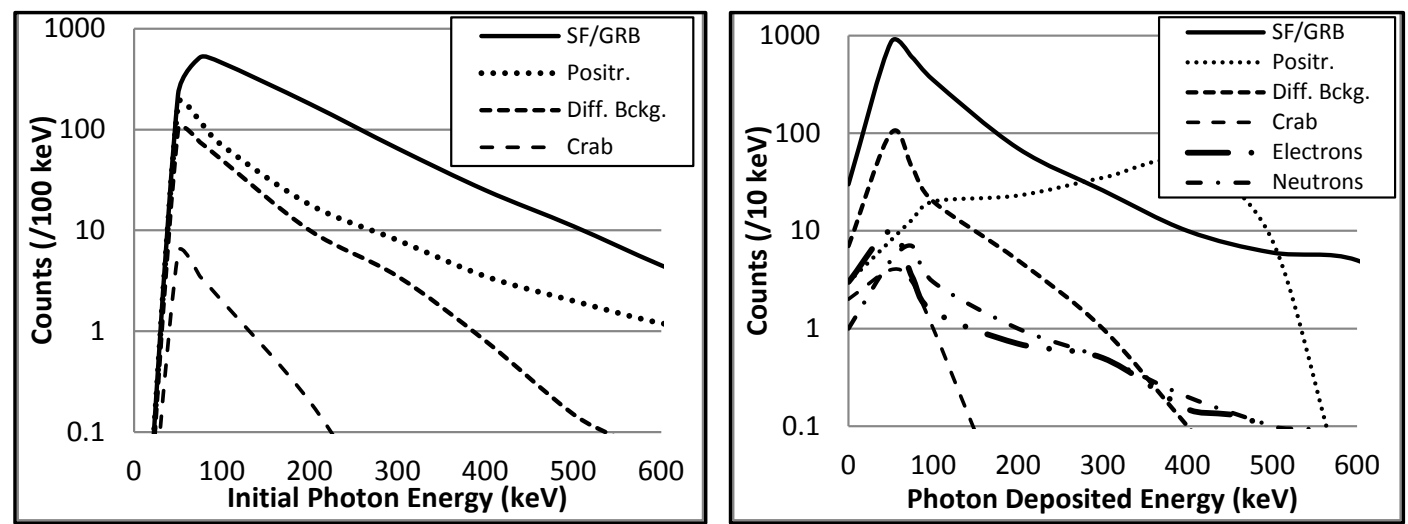

Figure 5. Example of POLAR instrument background modeling. Various sources are showed as a function of initial (left) and deposited (right) photon energy and compared with the main signal (typical SF or GRB). 

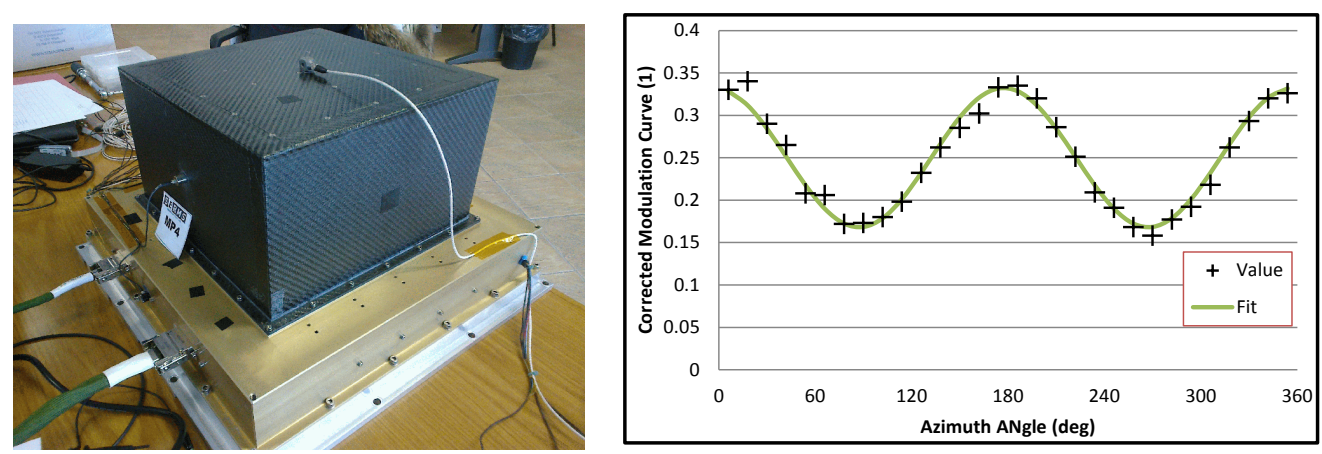

Figure 6. Photograph of the QM (left); Modulation curve and data fit (right).

Figure 6 (right). The fit value was $\mu_{100}=0.33$ as expected from the modeling. To date all environmental tests of the QM were successfully passed. The FMS is already in China for pre-integration tests while the FM is subjected to the final qualification in Europe. Its delivery is planned for July 2015. Shortly after the full preparations for the launch by mid of 2016 will begin, starting with the integration to the Chinese Space Station TG2.

\section{Prediction for Solar Flare Detection}

TG2 will be flying during a decreasing activity of the solar cycle 24 . This cycle turned out to be the weakest one for the last 100 years. Despite of it there is a big chance to detect a sufficient number of X-class solar flares and achieve major advancements in the polarization determination. Our predictions rely on the comparative studies between cycle 23 and 24. Both, the expected numbers of sun spots as well as forecasted intensity of the current cycle were used for analysis. Predicted period covers two years of observations starting from the middle of 2016. Estimated number of the X-class flares to be seen by POLAR is between 11 and 21. Spectra of the flares were assumed to have a broken power law behavior with an index range $(2.0-2.5)$ for energies up to $100 \mathrm{keV}$ and (3.0 - 4.0) for energies from $100 \mathrm{keV}$ to $450 \mathrm{keV}$. The number of coincidence events for photon energies from $50 \mathrm{keV}$ to $450 \mathrm{keV}$ reached levels of 120,000 for time intervals between $120 \mathrm{~s}$ and $240 \mathrm{~s}$ (with the total flare duration of about 1 hour). These predictions show that polarization determination with the accuracy better than $5 \%$ is fully possible.

\section{Summary and Conclusions}

New hard X-ray polarimeter POLAR for space-based observations was manufactured and currently undergoes final environmental and calibration tests. It will be installed on board of the Chinese space station TG2 with an anticipated launch date in mid of 2016. The novel measurement principle of POLAR utilizes Compton scattering with coincidence detections between two fast, light plastic scintillator bars. Its large field of view ( $1 / 3$ of the sky), the matrix of $40 \times 40$ scintillating bars with the effective area $A_{\text {eff }}$ of almost $200 \mathrm{~cm}^{2}$ and big modulation factor (maximum $\mu_{100} \approx 0.35$ ) enable the instrument for the first time to conduct polarization measurements on statistically significant sample GRBs and SFs. Even in the period of decreasing solar activity of the current cycle the estimated number of observed X-class solar flares may reach a value of few tens. For most of them the levels of accuracy will be better than 5\% finally enabling to pin down acceleration mechanisms and photon emission processes and conditions.

\section{References}

[1] Jeffrey, N.L.S. and Kontar, E.P. (2011) Spatially Resolved Hard X-Ray Polarization in Solar Flares: Effects of Compton Scattering and Bremsstrahlung. A\&A, 536, 1-15.

[2] Boggs, S.E., Coburn, W. and Kalemci, E. (2006) Gamma-Ray Polarimetry of Two X-Class Solar Flares. Astrophys. J. 638, 1129-1139. http://dx.doi.org/10.1086/498930

[3] Suarez-Garcia, E., et al. (2006) X-Ray Polarization of Solar Flares Measured with Rhessi. Solar Phys., 239, $149-172$. http://dx.doi.org/10.1007/s11207-006-0268-1

[4] Hajdas, W. and Suarez-Garcia, E. (2013) Polarimetry at High Energies. In: Huber, M.C.E., et al., Eds., Observing Photons in Space, 2nd Edition, 599-617. http://dx.doi.org/10.1007/978-1-4614-7804-1 34 
[5] Kanbach, G. and The MEGA Collaboration (2005) Development and Calibration of the Tracking Compton/Pair Telescope MEGA. NIM-A, 541, 310-322. http://dx.doi.org/10.1016/j.nima.2005.01.071

[6] Pearce, M., et al. (2011) PoGOLite-A Balloon-Borne X-Ray Polarimeter. 13th ICATPP Conference on Astroparticle, Particle, Space Physics and Detectors for Physics Applications, VillaOlma, Como, 6 October 2011.

[7] Produit, N., et al. (2005) POLAR, a Compact Detector for Gamma-Ray Bursts Photon Polarization Measurements. NIM, A550, 616-625. http://dx.doi.org/10.1016/j.nima.2005.05.066

[8] Suarez Garcia, E. (2010) X-Ray Polarization: RHESSI Results and POLAR Prospects. Ph.D. Thesis, Universitte de Geneve. 